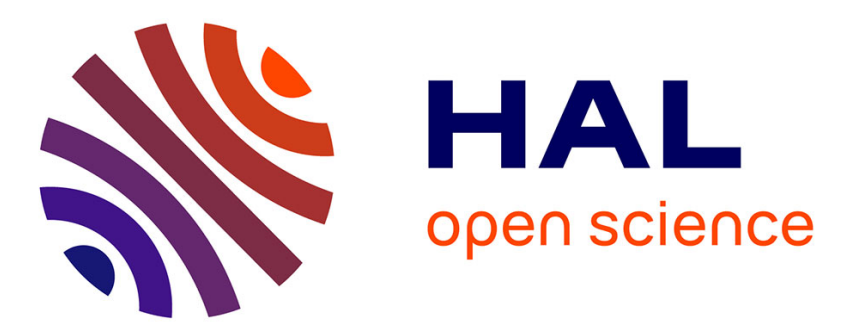

\title{
Efficient multicomponent synthesis of 2-aminopyridines catalysed by basic mesoporous materials
} Fatiha Nouali, Zahira Kibou, Bouhadjar Boukoussa, Noureddine

Choukchou-Braham, Abdelkader Bengueddach, Didier Villemin, Rachida Hamacha

\section{To cite this version:}

Fatiha Nouali, Zahira Kibou, Bouhadjar Boukoussa, Noureddine Choukchou-Braham, Abdelkader Bengueddach, et al.. Efficient multicomponent synthesis of 2-aminopyridines catalysed by basic mesoporous materials. Research on Chemical Intermediates, 2020, 46, pp.3179-3191. 10.1007/s11164-02004144-5 . hal-02880243

HAL Id: hal-02880243

https://hal-normandie-univ.archives-ouvertes.fr/hal-02880243

Submitted on 5 Jul 2020

HAL is a multi-disciplinary open access archive for the deposit and dissemination of scientific research documents, whether they are published or not. The documents may come from teaching and research institutions in France or abroad, or from public or private research centers.
L'archive ouverte pluridisciplinaire HAL, est destinée au dépôt et à la diffusion de documents scientifiques de niveau recherche, publiés ou non, émanant des établissements d'enseignement et de recherche français ou étrangers, des laboratoires publics ou privés. 


\title{
Efficient multicomponent synthesis of 2-aminopyridines catalysed by basic mesoporous materials
}

\author{
Fatiha Nouali $^{1} \cdot$ Zahira Kibou $^{1,2}$. Bouhadjar Boukoussa ${ }^{2,3}$. \\ Noureddine Choukchou-Braham ${ }^{1}$. Abdelkader Bengueddach ${ }^{3}$. Didier \\ Villemin $^{4} \cdot$ Rachida Hamacha ${ }^{3}$
}

${ }^{1}$ Laboratoire de Catalyse et Synthèse en Chimie Organique, Faculté des

Sciences, Université de Tlemcen, BP 119, 13000 Tlemcen, Algeria

${ }^{2}$ Centre Universitaire de Ain Témouchent, Institut des Sciences et de la Technologie, BP 284, 46000 Ain Témouchent, Algeria

${ }^{3}$ Laboratoire de Chimie des Matériaux L.C.M, Université d'Oran 1, BP 1524 El-Mnaouer, 31000 Oran, Algeria

${ }^{4}$ Laboratoire de Chimie Moléculaire et Thioorganique, UMR CNRS

6507, INC3M, FR 3038, ENSICAEN et Université de Caen Basse-

Normandie, 14050 Caen, France

\begin{abstract}
An efficient and green procedure for the synthesis of 2aminopyridines derivatives via four-component reaction of acetophenone, malononitrile, triethoxymethane and different primary amines has been developed using different surfactant-containing mesoporous catalysts. The advantages of this approach are easy operational method, high product yields and reusable catalysts without losing their catalytic activity.
\end{abstract}

Graphical abstract 


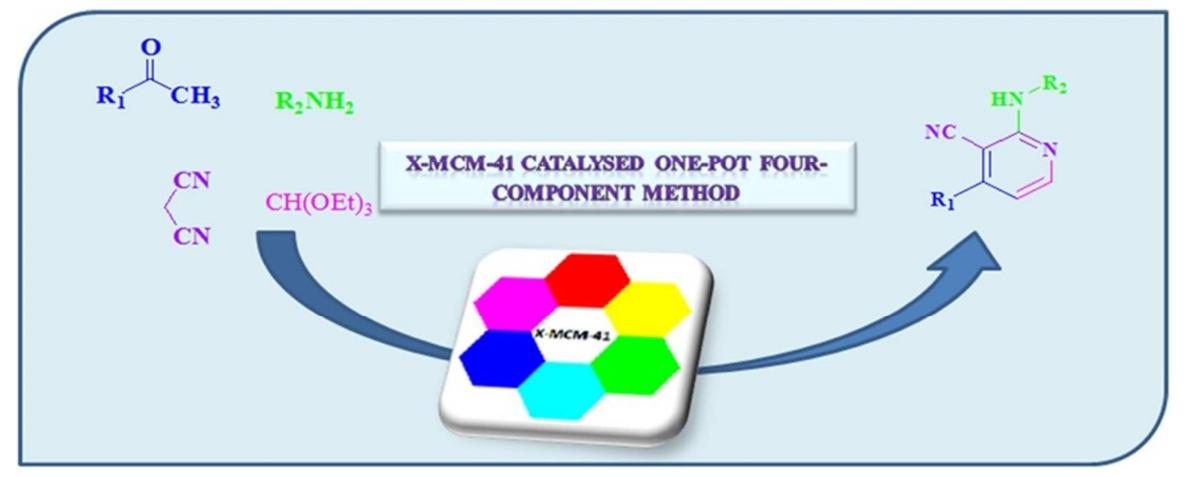

Keywords Multicomponent reactions of 2-aminopyridines synthesis . Solvent-free · Mesoporous materials · MCM-41 - Catalyst reuse

\section{Introduction}

Multicomponent reactions (MCRs) have received considerable attention and are known as some of the most important reactions in organic and medicinal chem- istry [1-11]. One-pot multicomponent reactions are the useful path for the fac- ile synthesis of heterocycles with a biological value [12-15]. In recent years, the challenge in organic synthesis has been the development of new MCRs using catalytic systems as well as MCM-41, clean, environmentally benign, efficient basic recyclable catalysts for the synthesis of heterocyclic compounds [16-18]. The structures of 2aminopyridines and its derivatives are a class of nitrogen heterocyclic compounds that have drawn much attention, due to their biological and pharmaceutical activities [19-24]. The development of clean synthetic routes towards nitrogen-containing heterocycles is of great importance to both synthetic and medicinal chemists [2527]. So, it is important to find simple and convenient procedures for the synthesis of 2-aminopyridines with different substitutes in their moiety, with the aim of obtaining some novel heterocyclic compounds with potentially enhanced properties. 
In the continuation of our research program on the development of green methodologies in the synthesis of biological important compounds using readily available, inexpensive and environmentally friendly catalysts [28-34], we wish to report in this work a new multicomponent reaction of 2-aminopyridines using mesoporous catalysts.

In the last decades, a particular interest was set on the use of the mesoporous silica in catalysis and adsorption [35-46], due to their higher specific surface areas and pore size compared to the zeolites. These catalysts may be easily recov- ered at the end of a reaction and can be reused in several cycles. Among these materials, the mesoporous silica MCM-41 has known very wide applications due to its bi-dimensional structure and its ordered pores [47, 48]. The mesoporous silica MCM-41 has been applied in several catalytic reactions as acid/base and/ or redox catalysts. However, due to the surface of the mesoporous silica MCM-41 containing only the silanol groups, several efforts have been made in order to activate its surface and make it useful in basic catalysis.

Among the most used methods for modifying the surface of the mesoporous silica, there is the dispersion of the metal oxides, the dispersion of the polymers having a basic character and the functionalization by amine groups. However, some of these methods can affect the structural and textural properties of the modified material. A particular interest has been devoted to the use of mesoporous silica containing its own surfactant without any modification, the non-calcined mesoporous silica with a strong basicity from the siloxy group bonded with $\mathrm{CTA}^{+}$[47]. These attractive cata- lysts have also been tested in different reactions which require basic catalysts such as Knoevenagel and Claisen-Schmidt condensations [48], Michael additions-cycloaddition reactions of $\mathrm{CO}_{2}$ with epoxides [48], cycloaddition of 4-nitrophenyl azide [34] and condensation of acetophenone with ethyl cyanoacetate [48]. Herein, we report a facile and rapid one-pot four-component route to the synthesis of 3-cyano-2-aminopyridines in the presence or absence of Al, Ga or Si-MCM-41 as-synthesized catalysts under free solvent conditions. The comparative studies have been performed between homogeneous and heterogeneous catalysis for the synthesis of 2-aminopyridines derivatives. 


\section{Experimental}

\section{Materials and physical measurements}

All products were prepared in our laboratory and analysed by spectroscopic methods. The melting points were measured using a Bank Kofler Heizbank apparatus standard WME 50-260 ${ }^{\circ} \mathrm{C}$ without particular correction. IR spectra were performed on solid samples using a Fourier transform PerkinElmer Spectrum with ATR accessory. Only significant absorptions are listed. The ${ }^{1} \mathrm{H}$ and ${ }^{13} \mathrm{C}$ NMR spectra were recorded on a Bruker AC 400 spectrometers at 400 and $100 \mathrm{MHz}$, respectively. Samples were recorded in $\mathrm{CDCl}_{3}$ solutions using TMS as an internal standard. The chemical shifts are expressed in $\delta$ units ( $\mathrm{ppm})$ and quoted downfield from TMS. The multiplicities are reported as: $s$, singlet; $d$, doublet; $t$, triplet; $q$, quartet; $m$, multiplet (Scheme 1).

\section{Synthesis}

\section{General synthesis of 2-aminopyridines without catalyst}

A mixture of acetophenone $(10 \mathrm{mmol}), 10 \mathrm{mmol}$ of malononitrile, $10 \mathrm{mmol}$ of triethoxymethane and primary amine $(10 \mathrm{mmol})$ was heated for $3 \mathrm{~h}$ at $100{ }^{\circ} \mathrm{C}$. After the completion of the reaction, the residue was purified by column chromatography over silica gel using a mixture of $n$ hexane-EtOAc (8:1) as the eluent to give desired compounds.

\section{General synthesis of 2-aminopyridines with catalyst}

A mixture of equimolar amount of the four compounds: acetophenone; malononitrile, triethoxyethane and benzylamine with $0.001 \mathrm{~mol}$ of (X-MCM-41), was stirred at room temperature without solvent. After the completion of the reaction, the catalyst was separated by filtration.

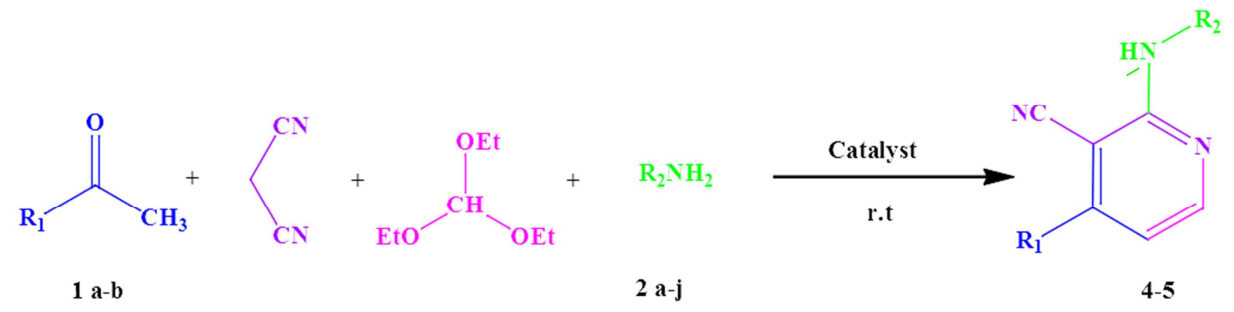

1a: $\mathrm{R}=\mathrm{Ph}$

1b: $\mathrm{R}=4-\mathrm{CH}_{4}-\mathrm{Ph}$

Scheme.1. Four-component reaction of 2-aminopyridines derivatives 
2-(Benzylamino)-4-phenylnicotinonitrile $\mathbf{4}_{\mathbf{a}}$ White solid, $\mathrm{mp} 95{ }^{\circ} \mathrm{C}$; IR $v_{\max }$ (neat/ $\left.\mathrm{cm}^{-1}\right)$ : $1575(\mathrm{C}=\mathrm{C}) ; 1531(\mathrm{C}=\mathrm{C}) ; 2215(\mathrm{CN}) ; 3361(\mathrm{NH}) ; \mathrm{NMR}{ }^{1} \mathrm{H}\left(\mathrm{CDCl}_{3}\right) \delta_{\mathrm{H}} \mathrm{ppm}$ : $4.6\left(2 \mathrm{H}, d, \mathrm{~J}_{\mathrm{H}-\mathrm{H}}=5.2 \mathrm{~Hz}, \mathrm{NH}-\mathrm{CH}_{2}\right) ; 5.60\left(1 \mathrm{H}, \mathrm{t}, \mathrm{J}_{\mathrm{H}-\mathrm{H}}=4.6 \mathrm{~Hz}, \mathrm{NH}-\mathrm{CH}_{2}\right)$;

$6.69\left(1 \mathrm{H}, d, \mathrm{~J}_{\mathrm{H}-\mathrm{H}}=4.6 \mathrm{~Hz}, \mathrm{CH}=\mathrm{CH}\right) ; 7.28-7.30\left(5 \mathrm{H}, \mathrm{m}, \mathrm{H}_{\text {arom }}\right) ; 7.46-7.58(5 \mathrm{H}, \mathrm{m}$, $\left.\mathrm{H}_{\text {arom }}\right) ; 8.30\left(1 \mathrm{H}, d, \mathrm{~J}_{\mathrm{H}-\mathrm{H}}=4.6 \mathrm{~Hz}, \mathrm{CH}=\mathrm{CH}\right) ; \mathrm{RMN}{ }^{13} \mathrm{C}\left(\mathrm{CDCl}_{3}\right) \delta_{\mathrm{C}}: 45.6$; 90.1; 113.1;116.9; 128.7-129.8; 136.7; 152; 154.50; 159.2; EIMS $\mathrm{m} / \mathrm{z}$ (\% relative abundance): 286 ( $\left.\mathrm{M}^{+} \mathrm{H}, 100\right), 208$ (23), 91 (93); HRMS (ESI-QTOF): Calcd for: $\mathrm{C}_{19} \mathrm{H}_{16} \mathrm{~N}_{3}$ M+ H 286,1339; Found: 286,1345.

2-(Cyclohexylamino)-4-phenylnicotinonitrile $\mathbf{4}_{\mathbf{b}}$ Yellow solid, mp $189{ }^{\circ} \mathrm{C}$; IR $v_{\max }$ $\left(\right.$ neat $\left./ \mathrm{cm}^{-1}\right): 1572(\mathrm{C}=\mathrm{C}) ; 1533(\mathrm{C}=\mathrm{C}) ; 2217(\mathrm{CN}) ; 3362(\mathrm{NH}) ; \mathrm{RMN}^{1} \mathrm{H}\left(\mathrm{CDCl}_{3}\right)$ 1.49-1.75 (m, 2H, $\left.-\left(\mathrm{CH}_{2}\right)_{4}-\right) ; 1.75(1 \mathrm{H}, \mathrm{q}, \mathrm{CH}-\mathrm{NH}) ; 5.22(1 \mathrm{H}, \mathrm{s}, \mathrm{NH}) ; 6.33(1 \mathrm{H}, \mathrm{d}$, $\left.J_{H-H}=5.8 \mathrm{~Hz}, \mathrm{CH}=\mathrm{CH}-\mathrm{N}\right) ; 7.325-7.56\left(5 \mathrm{H}, \mathrm{m}, \mathrm{H}_{\mathrm{arom}}\right) ; 8.26\left(1 \mathrm{H}, \mathrm{d}, J_{H-H}=5.7 \mathrm{~Hz}\right.$, $\mathrm{CH}=\mathrm{CH}-\mathrm{N})$; $\mathrm{RMN}{ }^{13} \mathrm{C}\left(\mathrm{CDCl}_{3}\right) \delta_{\mathrm{C}}: 11.1 ; 23.6 ; 46.5 ; 88.3 ; 105.1 ; 117.0 ; 127.3-$ 129.3;152.3; 155.3; 156.2; EIMS m/z (\% relative abundance): $277\left(\mathrm{M}^{+} \mathrm{H}, 100\right),(19)$, HRMS (ESI-QTOF): Calcd for: $\mathrm{C}_{18} \mathrm{H}_{20} \mathrm{~N}_{3} \mathrm{M}+\mathrm{H} 277,1324$; Found: 277,1122.

2-(Hexylamino)-4-phenylnicotinonitrile $\mathbf{4}_{\mathbf{c}}$ Yellow solid, mp $120{ }^{\circ} \mathrm{C}$; IR $v_{\max }$ (neat/ $\left.\mathrm{cm}^{-1}\right)$ : $1571(\mathrm{C}=\mathrm{C}) ; 1532(\mathrm{C}=\mathrm{C}) ; 2215(\mathrm{CN}) ; 3360(\mathrm{NH}) ; \mathrm{RMN}^{1} \mathrm{H}\left(\mathrm{CDCl}_{3}\right) \delta_{\mathrm{H}}$ : $0.98\left(3 \mathrm{H}, \mathrm{t}, \mathrm{J}_{\mathrm{H}-\mathrm{H}}=7.2 \mathrm{~Hz},-\left(\mathrm{CH}_{2}\right)_{5}-\mathrm{CH}_{3}\right) ; 1.29\left(2 \mathrm{H}, \mathrm{m},-\mathrm{N}-\mathrm{CH}_{2}-\mathrm{CH}_{2}-\mathrm{CH}_{2}-\mathrm{CH}_{3}\right)$; $1.30\left(2 \mathrm{H}, \mathrm{m}, \mathrm{N}-\mathrm{CH}_{2}-\mathrm{CH}_{2}-\mathrm{CH}_{2} \mathrm{CH}_{2}-\mathrm{CH}_{2}-\mathrm{CH}_{3}\right) ; 1.33\left(2 \mathrm{H}, \mathrm{t}, J_{H-H}=4.20 \mathrm{~Hz} \mathrm{~N}-\right.$ $\left.\mathrm{CH}_{2}-\mathrm{CH}_{2} \mathrm{CH}_{2}-\mathrm{CH}_{2}-\mathrm{CH}_{2}-\mathrm{CH}_{3}\right), 1.52\left(2 \mathrm{H}, \mathrm{t}, \mathrm{J}_{\mathrm{H}-\mathrm{H}}=4.20 \mathrm{~Hz} \mathrm{~N}-\mathrm{CH}_{2}-\mathrm{CH}_{2} \mathrm{CH}_{2}-\right.$ $\left.\mathrm{CH}_{2}-\mathrm{CH}_{2}-\mathrm{CH}_{3}\right), 3.53\left(2 \mathrm{H}, \mathrm{t}, \mathrm{J}_{\mathrm{H}}-\mathrm{H}=4.20 \mathrm{~Hz}, \mathrm{~m}, \mathrm{~N}-\mathrm{CH}_{2}-\mathrm{CH}_{2} \mathrm{CH}_{2}-\mathrm{CH}_{2}-\mathrm{CH}_{2}-\mathrm{CH}_{3}\right)$; $5.36\left(1 \mathrm{H}, \mathrm{t}, J_{H-H}=4.20 \mathrm{~Hz}, \mathrm{NH}\right) ; 6.63\left(1 \mathrm{H}, \mathrm{d}, J_{H-H}=5.8 \mathrm{~Hz}, \mathrm{CH}=\mathrm{CH}-\mathrm{N}\right) ; 7.35-7.56(5 \mathrm{H}$, $\left.\mathrm{m}, \mathrm{H}_{\text {arom }}\right) ; 8.31\left(1 \mathrm{H}, \mathrm{d}, J_{H-H}=5.7 \mathrm{~Hz}, \mathrm{CH}=\mathrm{CH}-\mathrm{N}\right) ; \mathrm{RMN}{ }^{13} \mathrm{C}\left(\mathrm{CDCl}_{3}\right) \delta_{\mathrm{C}}$ : $14.1 ; 22.6 ; 26.5 ; 32.6 ; 44.5 ; 88.3 ; 105.1 ; 113.4 ; 117 ; 126.2-127.5 ; 136.4 ; 152.3155 .3$; 155.3; EIMS m/z (\% relative abundance): $280\left(\mathrm{M}^{+} \mathrm{H}, 100\right), 200$ (19), HRMS (ESI- QTOF): Calcd for: $\mathrm{C}_{18} \mathrm{H}_{22} \mathrm{~N}_{3} \mathrm{M}+\mathrm{H} 279,1422$; Found: 279,1345 .

2-(Propylamino)-4-phenylnicotinonitrile $\mathbf{4}_{\mathbf{d}}$ Yellow solid, mp $111{ }^{\circ} \mathrm{C}$; IR $v_{\max }$ (neat/ $\left.\mathrm{cm}^{-1}\right): 1583(\mathrm{C}=\mathrm{C}), 1554(\mathrm{C}=\mathrm{C}) ; 2219(\mathrm{CN}) ; 3383(\mathrm{NH}) ; \mathrm{RMN}^{1} \mathrm{H}\left(\mathrm{CDCl}_{3}\right): \delta_{\mathrm{H}} 1.01(3 \mathrm{H}, \mathrm{t}$, $\left.\mathrm{J}_{\mathrm{H}-\mathrm{H}}=7.4 \mathrm{~Hz},-\left(\mathrm{CH}_{2}\right)_{2}-\mathrm{CH}_{3}\right) ; 1.56\left(2 \mathrm{H}, \mathrm{m},-\mathrm{N}-\mathrm{CH}_{2}-\mathrm{CH}_{2}-\mathrm{CH}_{3}\right) ; 3.11(2 \mathrm{H}, \mathrm{m},-\mathrm{N}-$ $\left.\mathrm{CH}_{2}-\mathrm{CH}_{2}-\mathrm{CH}_{3}\right) ; 5.22\left(1 \mathrm{H}, \mathrm{t}, J_{H-H}=4.20 \mathrm{~Hz}, \mathrm{NH}\right) ; 6.64\left(1 \mathrm{H}, \mathrm{d}, J_{H-H}=5.8 \mathrm{~Hz}, \mathrm{CH}=\mathrm{CH}-\right.$ $\mathrm{N}) ; 7.325-7.56\left(5 \mathrm{H}, \mathrm{m}, \mathrm{H}_{\text {arom }}\right) ; 8.26\left(1 \mathrm{H}, \mathrm{d}, J_{H-H}=5.7 \mathrm{~Hz}, \mathrm{CH}=\mathrm{CH}-\mathrm{N}\right) ; \mathrm{RMN}^{13} \mathrm{C}$ $\left(\mathrm{CDCl}_{3}\right) \delta_{\mathrm{C}}: 11.1 ; 23.6 ; 46.5 ; 88.3 ; 105.1 ; 117 ; 127.3-129.3 ; 152.3 ; 155.3$;

156.2; EIMS m/z (\% relative abundance): $237\left(\mathrm{M}^{+} \mathrm{H}, 100\right),(20)$, HRMS (ESI- QTOF): Calcd for: $\mathrm{C}_{15} \mathrm{H}_{16} \mathrm{~N}_{3} \mathrm{M}+\mathrm{H} 237,1422$; Found: 237,1235.

2-(Butylamino)-4-phenylnicotinonitrile 4e White solid, mp $159{ }^{\circ} \mathrm{C}$; IR $v_{\max } \mathrm{cm}^{-1}$;

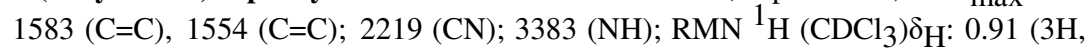
t, $\left.\mathrm{J}_{\mathrm{H}-\mathrm{H}}=7.2 \mathrm{~Hz},-\left(\mathrm{CH}_{2}\right)_{3}-\mathrm{CH}_{3}\right) ; 1.32\left(2 \mathrm{H}, \mathrm{m},-\mathrm{N}-\mathrm{CH}_{2}-\mathrm{CH}_{2}-\mathrm{CH}_{2}-\mathrm{CH}_{3}\right) ; 1.33(2 \mathrm{H}$, $\left.\mathrm{m}, \mathrm{N}-\mathrm{CH}_{2}-\mathrm{CH}_{2}-\mathrm{CH}_{2}-\mathrm{CH}_{3}\right) ; 2.64\left(2 \mathrm{H}, \mathrm{t}, \mathrm{J}_{\mathrm{H}-\mathrm{H}}=4.20 \mathrm{~Hz} \mathrm{~N}-\mathrm{CH}_{2}-\mathrm{CH}_{2}-\mathrm{CH}_{2}-\mathrm{CH}_{3}\right)$; $5.36\left(1 \mathrm{H}, \mathrm{t}, J_{H-H}=4.20 \mathrm{~Hz}, \mathrm{NH}\right) ; 6.63\left(1 \mathrm{H}, \mathrm{d}, J_{H-H}=5.8 \mathrm{~Hz}, \mathrm{CH}=\mathrm{CH}-\mathrm{N}\right) ; 7.35-7.56$ $\left(5 \mathrm{H}, \mathrm{m}, \mathrm{H}_{\mathrm{arom}}\right) ; 8.31\left(1 \mathrm{H}, \mathrm{d}, J_{H-H}=5.8 \mathrm{~Hz}, \mathrm{CH}=\mathrm{CH}-\mathrm{N}\right) ; \mathrm{RMN}^{13} \mathrm{C}\left(\mathrm{CDCl}_{3}\right) \delta_{\mathrm{C}}$ : $13.71\left(\mathrm{CH}_{3}\right) ; 21.3 ; 32 ; 41.5 ; 88.3 ; 113.42 ; 117.01 ; 126.25-129.86 ; 136.88 ; 152.08$; 155.34; 160.12. EIMS m/z (\% relative abundance): $251(\mathrm{M}+\mathrm{H}, 100),(19)$, HRMS (ESI-QTOF): Calcd for: $\mathrm{C}_{16} \mathrm{H}_{18} \mathrm{~N}_{3} \mathrm{M}+\mathrm{H} 251,1348$; Found: 251,1356 .

2-(Benzylamino)-4-p-tolylnicotinonitrile $\mathbf{5}_{\mathbf{a}}$ White solid, mp $189{ }^{\circ} \mathrm{C}$; IR $v_{\max }$ (neat/ $\left.\mathrm{cm}^{-1}\right): 1578,1533(\mathrm{C}=\mathrm{C}) ; 2217(\mathrm{CN}) ; 3363(\mathrm{NH}) ; \mathrm{RMN}{ }^{1} \mathrm{H}\left(\mathrm{CDCl}_{3}\right) \delta_{\mathrm{H}}: 2.41(3 \mathrm{H}, \mathrm{s}, \mathrm{Ph}-$ 
$\left.\mathrm{CH}_{3}\right) ; 4.68\left(2 \mathrm{H}, \mathrm{d}, J_{H-H}=4.6 \mathrm{~Hz}, \mathrm{NH}-\mathrm{CH}_{2}\right) ; 5.66\left(1 \mathrm{H}, \mathrm{t}, J_{H-H}=4.6 \mathrm{~Hz}, \mathrm{NH}-\mathrm{CH}_{2}\right)$; $6.70\left(1 \mathrm{H}, \mathrm{d}, J_{H-H}=4.6 \mathrm{~Hz}, \mathrm{CH}=\mathrm{CH}\right) ; 7.25-7.30\left(5 \mathrm{H}, \mathrm{m}, \mathrm{H}_{\mathrm{arom}}\right) ; 7.46-7.58(4 \mathrm{H}, \mathrm{m}$, $\left.\mathrm{H}_{\text {arom }}\right) ; 8.35\left(1 \mathrm{H}, \mathrm{d}, J_{H-H}=4.6 \mathrm{~Hz}, \mathrm{CH}=\mathrm{CH}\right) ; \mathrm{RMN}{ }^{13} \mathrm{C}\left(\mathrm{CDCl}_{3}\right) \delta_{\mathrm{C}}: 24.5\left(\mathrm{Ph}-\mathrm{CH}_{3}\right)$, $45.6\left(\mathrm{CH}_{2}\right)$; $91.1(\mathrm{C}=\mathrm{C}-\mathrm{CN}) ; 113.4(\mathrm{CH}=\mathrm{CH}) ; 116.8(\mathrm{CN}) ; 127.3-127.9\left(5 \times \mathrm{C}_{\text {arom }}\right) ; 128.1-$ $129.5\left(5 \mathrm{xC}_{\text {arom }}\right) ; 136\left(\mathrm{C}_{\text {arom }}\right) ; 139.2\left(\mathrm{C}_{\text {arom }}\right) ; 153.1(\mathrm{CH}=\mathrm{CH})$;

$154.6(\mathrm{C}=\mathrm{C}-\mathrm{CN}) ; 160.1(\mathrm{~N}=\mathrm{C}-\mathrm{NH}) ;$ EIMS m/z (\% relative abundance $): 300\left(\mathrm{M}^{+} \mathrm{H}\right.$, 80), 150 (25), HRMS (ESI-QTOF): Calcd for: $\mathrm{C}_{20} \mathrm{H}_{19} \mathrm{~N}_{3} \mathrm{M}^{+} \mathrm{H}$ 300,1244; Found: 300,1345 .

2-(Cyclohexylamino)-4-p-tolylnicotinonitrile $\mathbf{5}_{\mathbf{b}}$ Yellow solid, mp $189{ }^{\circ} \mathrm{C}$; IR $v_{\max }$ $\left(\right.$ neat $\left./ \mathrm{cm}^{-1}\right): 1575,1533(\mathrm{C}=\mathrm{C}) ; 2221(\mathrm{CN}) ; 3365(\mathrm{NH}) ; \mathrm{RMN}^{1} \mathrm{H}\left(\mathrm{CDCl}_{3}\right) \delta_{\mathrm{H}}: 1.48-$ $1.76\left(\mathrm{~m}, 2 \mathrm{H},-\left(\mathrm{CH}_{2}\right)_{4}-\right) ; 1.75(1 \mathrm{H}, \mathrm{q}, \mathrm{CH}-\mathrm{NH}) ; 2.35\left(3 \mathrm{H}, \mathrm{s},-\mathrm{CH}_{3}\right) ; 5.22(1 \mathrm{H}, \mathrm{s}$, $\mathrm{NH}) ; 6.32\left(1 \mathrm{H}, \mathrm{d}, J_{H-H}=5.8 \mathrm{~Hz}, \mathrm{CH}=\mathrm{CH}-\mathrm{N}\right) ; 7.325-7.56\left(5 \mathrm{H}, \mathrm{m}, \mathrm{H}_{\text {arom }}\right) ; 8.26(1 \mathrm{H}, \mathrm{d}$, $\left.J_{H-H}=5.7 \mathrm{~Hz}, \mathrm{CH}=\mathrm{CH}-\mathrm{N}\right) ; \mathrm{RMN}{ }^{13} \mathrm{C}\left(\mathrm{CDCl}_{3}\right) \delta_{\mathrm{C}}: 11.1 ; 23.6 ; 24.4 ; 46.5 ; 88.3$; $105.1 ; 117 ; 127.3-129.3 ; 152.4 ; 155.3 ; 156.2$; EIMS m/z (\% relative abundance): $291\left(\mathrm{M}^{+} \mathrm{H}, 100\right)$, (19), HRMS (ESI-QTOF): Calcd for: $\mathrm{C}_{19} \mathrm{H}_{22} \mathrm{~N}_{3} \mathrm{M}+\mathrm{H} 291,1224$; Found: 291,1155 .

2-(Hexylamino)-4-p-tolylnicotinonitrile $\mathbf{5}_{\mathbf{c}}$ Yellow solid, $\mathrm{mp} 220{ }^{\circ} \mathrm{C}$; IR $v_{\max }($ neat/ $\left.\mathrm{cm}^{-1}\right): 1572,1532(\mathrm{C}=\mathrm{C}) ; 2219(\mathrm{CN}) ; 3363(\mathrm{NH}) ; \mathrm{RMN}^{1} \mathrm{H}\left(\mathrm{CDCl}_{3}\right) \delta_{\mathrm{H}}: 0.99\left(3 \mathrm{H}, \mathrm{t}, \mathrm{J}_{\mathrm{H}-\mathrm{H}}=\right.$ $\left.7.2 \mathrm{~Hz},-\left(\mathrm{CH}_{2}\right)_{5}-\mathrm{CH}_{3}\right) ; 1.27\left(2 \mathrm{H}, \mathrm{m},-\mathrm{N}-\mathrm{CH}_{2}-\mathrm{CH}_{2}-\mathrm{CH}_{2}-\mathrm{CH}_{3}\right) ; 1.30\left(2 \mathrm{H}, \mathrm{m}, \mathrm{N}-\mathrm{CH}_{2}-\right.$ $\left.\mathrm{CH}_{2}-\mathrm{CH}_{2} \mathrm{CH}_{2}-\mathrm{CH}_{2}-\mathrm{CH}_{3}\right) ; 1.32\left(2 \mathrm{H}, \mathrm{t}, \mathrm{J}_{\mathrm{H}}-\mathrm{H}=4.20 \mathrm{~Hz} \mathrm{~N}-\mathrm{CH}_{2}-\mathrm{CH}_{2} \mathrm{CH}_{2}-\mathrm{CH}_{2}-\mathrm{CH}_{2}-\right.$ $\left.\mathrm{CH}_{3}\right), 1.52\left(2 \mathrm{H}, \mathrm{t}, \mathrm{J}_{\mathrm{H}-\mathrm{H}}=4.20 \mathrm{~Hz} \mathrm{~N}-\mathrm{CH}_{2}-\mathrm{CH}_{2} \mathrm{CH}_{2}-\mathrm{CH}_{2}-\mathrm{CH}_{2}-\mathrm{CH}_{3}\right)$,

$2.35\left(\left(3 \mathrm{H}, \mathrm{s},-\mathrm{CH}_{3}\right) ; 3.42\left(2 \mathrm{H}, \mathrm{t}, J_{H-H}=4.20 \mathrm{~Hz}, \mathrm{~m}, \mathrm{~N}-\mathrm{CH}_{2}-\mathrm{CH}_{2} \mathrm{CH}_{2}-\mathrm{CH}_{2}-\mathrm{CH}_{2}-\right.\right.$ $\left.\mathrm{CH}_{3}\right) ; 5.29\left(1 \mathrm{H}, \mathrm{t}, J_{H-H}=4.20 \mathrm{~Hz}, \mathrm{NH}\right) ; 6.63\left(1 \mathrm{H}, \mathrm{d}, J_{H-H}=5.8 \mathrm{~Hz}, \mathrm{CH}=\mathrm{CH}-\mathrm{N}\right)$; $7.33-7.52\left(5 \mathrm{H}, \mathrm{m}, \mathrm{H}_{\text {arom }}\right) ; 8.28\left(1 \mathrm{H}, \mathrm{d}, J_{H-H}=5.7 \mathrm{~Hz}, \mathrm{CH}=\mathrm{CH}-\mathrm{N}\right) ; \mathrm{RMN}{ }^{13} \mathrm{C}$ $\left(\mathrm{CDCl}_{3}\right) \delta_{\mathrm{C}}: 14.1 ; 22.61 ; 24.3 ; 26.5 ; 32.6 ; 43.4 ; 88.2 ; 105.1 ; 113.3 ; 117 ; 127.5-$ 136.4;152.3; 155.3; 155.6; EIMS m/z (\% relative abundance): $293\left(\mathrm{M}^{+} \mathrm{H}, 100\right), 200$ (19), HRMS (ESI-QTOF): Calcd for: $\mathrm{C}_{19} \mathrm{H}_{24} \mathrm{~N}_{3} \mathrm{M}+\mathrm{H}$ 293,1322; Found: 293,1115.

2-(Propylamino)-4-p-tolylnicotinonitrile $\mathbf{5}_{\mathbf{d}}$ Yellow solid, mp $181{ }^{\circ} \mathrm{C}$; IR $v_{\max }$ (neat/ $\left.\mathrm{cm}^{-1}\right): 1570,1531(\mathrm{C}=\mathrm{C}) ; 2217(\mathrm{CN}) ; 3365(\mathrm{NH}) ; \mathrm{NMR}^{1} \mathrm{H}\left(\mathrm{CDCl}_{3}\right) \delta_{\mathrm{H}} \mathrm{ppm} 1.49$ $1.75\left(\mathrm{~m}, 2 \mathrm{H},-\left(\mathrm{CH}_{2}\right) 4^{-}\right) ; 1.75(1 \mathrm{H}, \mathrm{q}, \mathrm{CH}-\mathrm{NH}) ; 2.35\left(3 \mathrm{H}, \mathrm{s},-\mathrm{CH}_{3}\right) ; 5.31(1 \mathrm{H}, \mathrm{s}$, $\mathrm{NH}) ; 6.29\left(1 \mathrm{H}, \mathrm{d}, J_{H-H}=5.8 \mathrm{~Hz}, \mathrm{CH}=\mathrm{CH}-\mathrm{N}\right) ; 7.25-7.49\left(5 \mathrm{H}, \mathrm{m}, \mathrm{H}_{\mathrm{arom}}\right) ; 8.23(1 \mathrm{H}, \mathrm{d}$, $\left.J_{H-H}=5.7 \mathrm{~Hz}, \mathrm{CH}=\mathrm{CH}-\mathrm{N}\right) ; \mathrm{RMN}{ }^{13} \mathrm{C}\left(\mathrm{CDCl}_{3}\right) \delta_{\mathrm{C}}: 10.1 ; 23.3 ; 24.3 ; 45.4 ; 88.4$; $105.1 ; 117 ; 127.3-129.3 ; 151.3 ; 155.3 ; 156.5 ;$ EIMS $\mathrm{m} / \mathrm{z}$ (\% relative abundance): $251\left(\mathrm{M}^{+} \mathrm{H}, 100\right)$, (19), HRMS (ESI-QTOF): Calcd for: $\mathrm{C}_{16} \mathrm{H}_{18} \mathrm{~N}_{3} \mathrm{M}+\mathrm{H} 251,1324$; Found: 251,1223. 
2-(Butylamino)-4-p-tolylnicotinonitrile 5e White solid, mp $147{ }^{\circ} \mathrm{C}$; IR $v_{\max } \mathrm{cm}^{-1}$ : $1582(\mathrm{C}=\mathrm{C}), 1553(\mathrm{C}=\mathrm{C}) ; 2222(\mathrm{CN}) ; 3381(\mathrm{NH}) ; \mathrm{RMN}^{1} \mathrm{H}\left(\mathrm{CDCl}_{3}\right) \delta_{\mathrm{H}}: 0,91(3 \mathrm{H}$, t, $\left.J_{H-H}=7.2 \mathrm{~Hz},-\left(\mathrm{CH}_{2}\right)_{3}-\mathrm{CH}_{3}\right) ; 1.33\left(2 \mathrm{H}, \mathrm{m},-\mathrm{N}-\mathrm{CH}_{2}-\mathrm{CH}_{2}-\mathrm{CH}_{2}-\mathrm{CH}_{3}\right) ; 1.34(2 \mathrm{H}$, $\left.\mathrm{m}, \mathrm{N}-\mathrm{CH}_{2}-\mathrm{CH}_{2}-\mathrm{CH}_{2}-\mathrm{CH}_{3}\right) ; 2.40\left(3 \mathrm{H}, \mathrm{s}, \mathrm{Ph}-\mathrm{CH}_{3}\right) ; 2.66\left(2 \mathrm{H}, \mathrm{t}, J_{H-H}=4.8 \mathrm{~Hz}, \mathrm{~N}-\right.$ $\left.\mathrm{CH}_{2}-\mathrm{CH}_{2}-\mathrm{CH}_{2}-\mathrm{CH}_{3}\right) ; 5.34\left(1 \mathrm{H}, \mathrm{t}, J_{H-H}=4.8 \mathrm{~Hz}, \mathrm{NH}\right) ; 6.61\left(1 \mathrm{H}, \mathrm{d}, J_{H-H}=5.9 \mathrm{~Hz}\right.$, $\mathrm{CH}=\mathrm{CH}-\mathrm{N}) ; 7.36-7.58\left(5 \mathrm{H}, \mathrm{m}, \mathrm{H}_{\text {arom }}\right) ; 8.27\left(1 \mathrm{H}, \mathrm{d}, J_{H-H}=5.9 \mathrm{~Hz}, \mathrm{CH}=\mathrm{CH}-\mathrm{N}\right) ; \mathrm{RMN}$ ${ }^{13} \mathrm{C}\left(\mathrm{CDCl}_{3}\right) \delta_{\mathrm{C}}: 13.5 ; 21.33 ; 24.51 ; 33.5 ; 42.4 ; 87.9 ; 113.6 ; 117.51 ; 126.3-$ $130.1 ; 137.9\left(\mathrm{C}_{\text {arom }}\right) ; 153.1(\mathrm{CH}=\mathrm{CH}-\mathrm{N}) ; 155.3(\mathrm{C}=\mathrm{C}-\mathrm{CN}) ; 159.5 ;$ EIMS m/z (\% relative abundance): $251\left(\mathrm{M}^{+} \mathrm{H}, 100\right),(19)$, HRMS (ESI-QTOF): Calcd for: $\mathrm{C}_{17} \mathrm{H}_{20} \mathrm{~N}_{3} \mathrm{M}+\mathrm{H} 265,1667$; Found: 251,1670.

\section{Results and discussion}

So we wish to report an efficient one-pot four-component method for the preparation of 2-aminopyridines. Moreover, to the best of our knowledge, this paper is the first report on the synthesis of some 2aminopyridines using as-synthesized mesoporous catalyst.

Initially, we have synthesized Al, Ga or Al-MCM-41 mesoporous materials con- taining $\mathrm{CTA}^{+}$by a direct method of crystallization according to the literature [37]. The detailed experimental procedures for the preparation of different as-synthesized mesoporous materials are well detailed and characterized in our previously published work [39].

Among the various methods developed for that purpose, multicomponent reactions involving domino processes answer perfectly to the green chemistry principles. In this context, and in connection with our recent investigation on the synthesis of nitrogen heterocycles under solvent-free conditions, firstly, we describe onepot four-component approach to synthesis 2-aminopyridines efficiently without catalyst. This approach is a process in which four easily accessible components are combined together in a single reaction 1 to produce a final product 4-5 (Table 1).

\section{Table 1}

However, using acetophenone with benzylamine gives good yields. After $3 \mathrm{~h}$ of heating at $100{ }^{\circ} \mathrm{C}$ all, the products $4-5$ were purified by column chromatography and isolated in yields ranging from 66 to 
$76 \%$ (Table 1). From this result, and in continuation of our interest in using solid catalysts in the synthesis of heterocycles, we describe here a simple and green strategy based on the new MCRs using MCM-41 as a powerful, recyclable and safe catalyst for the preparation of novel and known 3-cyano-2-aminopyridines 4-5 derivatives. Initially, a model reaction was conducted using acetophenone $(0.1 \mathrm{~mol})$, malononitrile $(0.1 \mathrm{~mol})$, triethoxymethane $(0.1 \mathrm{~mol})$ and benzylamine $(0.1 \mathrm{~mol})$ at room temperature in the presence of $0.001 \mathrm{~mol}$ (X-MCM-41) as catalyst under solvent-free conditions (Table 2); the product $\mathbf{4 a}$ is confirmed by their NMR, IR and MS analysis.

\section{Table 2}

The reaction proceeded best at ambient temperature without heating in the pres- ence of Si-MCM-41 to provide good yield $89 \%$ in a short time (15 min), and the results are summarized in Table 2, by against studying this reaction without heterogeneous catalyst leads to a product mixture.

\section{Table 1 Four-component reaction of 2-aminopyridines derivatives without catalyst}

To develop the scope of this reaction, and in order to evaluate the generality of this model reaction, we encouraged to extend this reaction to a variety of $\mathbf{1}_{\mathbf{a}-\mathbf{b}}$ with different primary amines $\mathbf{2} \mathbf{a}-\mathbf{j}$, so we have examined this reaction employing the optimized conditions, and the results are summarized in Table 3.

\section{Table 3}

It was clearly observed that the best results were obtained in the presence of Si-MCM-41 as catalyst at room temperature. Then, the generality of the procedure was evaluated using various primary amines under optimized reaction conditions. It was found that both using benzylamine as primary amine and Si- MCM-41 reacted well in this process to afford the corresponding products in 
good to excellent yields $92 \%$ (Table 3 ). So we can note that these MCRs are a selective synthesis of 2-aminopyridines using mesoporous catalyst.

The evolution of the yields is carried out in the following sequence: Si- MCM-41 > Al-MCM-41> Ga-MCM-41, and the assynthesized Si-MCM-41 pre- sents the best catalytic performance.

As shown in Fig. 1, all the catalysts have the same vibration bands of the as- synthesized MCM-41, the bands appeared between 2800 and $2900 \mathrm{~cm}^{-1}$ corresponding to the groups $-\mathrm{CH}_{2}$ and $-\mathrm{CH}_{3}$, and these bands also confirm the presence of surfactant $\left(\mathrm{CTA}^{+}\right)$linked with siloxy anions $\left(\equiv \mathrm{SiO}^{-}\right)$inside the pores of MCM-41which are considered as basic sites [36, 39, 48]. The choice of incorporating Al and $\mathrm{Ga}$ into the framework of Si-MCM-4 was to generate a bifunctional acid/base catalyst. But according to the catalytic reaction, the MCRs were well governed by the Si-MCM-41. This also shows that the reaction takes place with the basic catalyst Si- MCM-41 due to the presence of higher density of the siloxy groups [49-56].

It is also necessary to take into account the factor of the morphology which can affect the properties of catalyst. As shown in Fig. 2, it is clearly indicated that the catalysts Si- and Al-MCM-41 have a spherical morphology, while the catalyst Ga- MCM-41 has morphology in the form of aggregates probably due to the incorporation of the gallium leading subsequently to a slight destructuration of obtained catalyst.

\section{Fig. 2}

In order to examine the influence of amount for heterogeneous catalysts on MCRs, we have mixed 1a, $1 \mathrm{Eq}$ of acetophenone, $1 \mathrm{Eq}$ of malononitrile, $1 \mathrm{Eq}$ of tri- ethoxymethane and $1 \mathrm{Eq}$ of propylamine using different amounts of as-synthesized Si-MCM-41 (Fig. 3). T

Fig. 3

he use of $10 \mathrm{~mol} \%$ of as-synthesized Si-MCM-41 led to $80 \%$ yield for 25-min reaction time. We note in Fig. 1 that increasing the amount of cata- lyst has an impact on the reaction yield. Indeed, a 25-min reaction time led to a $89 \%$ yield using $20 \mathrm{~mol} \%$ (and $90 \%$ yield using $40 \mathrm{~mol} \%$ ) of as-synthesized Si-MCM-41. The yield obtained after 10 min of reaction in the presence of $50 \%$ of catalyst is about $98 \%$. 
Thus, the reaction time can be minimized due to an increase in catalytic amount of synthesized Si-MCM-41 catalyst and best results were obtained by per- forming the reaction in the presence of 50 mol\% of catalyst.

Furthermore, the reusability of Si-MCM-41 was also examined with the opti- mized experiment conditions of the model reaction for the synthesis of product 5a: acetophenone $(1 \mathrm{mmol})$, malononitrile (1 $\mathrm{mmol})$, triethoxymethane $(1 \mathrm{mmol})$ and $0.1 \mathrm{~mol}$ of benzylamine or cyclohexylamine in the presence of Si-MCM-41 (10 mol \%) at room temperature and reaction time for as-synthesized Si-MCM-41 being $15 \mathrm{~min}$. After completion of the reaction, the catalyst was isolated by simple filtration than the catalysts were filtered, washed with DMF and dried before use in the following cycles, and the results are represented in Fig. 4. This study demonstrated that SiMCM-41 could be effectively used as a recyclable catalyst for this new four-component reaction. The decrease in the activity of this catalyst during its reuse is strongly linked to the leaching of surfactant following its exposure on various occasions to treatment with solvents during cleaning. These results are in agreement with our work published previously $[56,57]$.

Fig. 4.

\section{Conclusion}

In conclusion, we have described a novel and efficient protocol for one-pot syn- thesis of 3-cyano-2-aminopyridines via a reaction between acetophenone derivative, malononitrile and triethoxymethane, using mesoporous materials as catalysts. The reaction time and product yields have been improved in the following order: Si-MCM$41>\mathrm{Al}-\mathrm{MCM}-41>\mathrm{Ga}-\mathrm{MCM}-41$; as-synthesized Si-MCM-41 is particularly efficient for this reaction at room temperature due to its higher number of ionic pairs $\mathrm{SiO}^{-}-\mathrm{CTA}^{+}$. Mild conditions, good to excellent yields, reusable catalysts and short reaction time are the advantages of the present method.

Acknowledgements We thank DGRSDT and the University of Tlemcen for funding this work. 


\section{References}

1. A. Dömling, Chem. Rev. 106, 17

(2006)

2. J. Zhu, H. Bienaymé, Multicomponent Reactions (Wiley-VCH,

Weinheim, 2005)

3. K. Kumaravel, G. Vasuki, Curr. Org. Chem. 13, 1820

(2009)

4. L.F. Tietze, Domino Reactions: Concepts for Efficient Organic Synthesis (Wiley$\mathrm{VCH}$, Wein- heim, 2014)

5. R.V.A. Orru, M. de Greef, Synthesis 10, 1471

(2003)

6. M. Umkeherer, C. Kalinski, J. Kolb, C. Burdack, Tetrahedron Lett. 47,

2391 (2006)

7. L.F. Tietze, G. Brasche, K. Gericke, Domino Reactions in Organic Synthesis (WileyVCH, Wein- heim, 2006)

8. A. Alizadeh, F. Mobahedi, A. Esmaili, Tetrahedron Lett. 47,

4469 (2006)

9. D. Pires, H. deOliveira, M.R.B. Pontel, J.S. Kazmierczak, R. Cargnelutti, D. Alve, R.J. Jacob, Beilstein J. Org. Chem. 14, 2789 (2018)

10. R. Vroemans, Y. Verhaegen, M.-T.D. Thi, W. Dehaen, Beilstein J. Org. Chem. 14, 2689 (2018)

11. S. Kamalifar, H. Kiyani, Res Chem Int. Med. 45, 5975

(2019)

12. N. Elders, R.F. Schmitz, F.J.J. Kanter, E. Ruijter, M.B. Groen, R.V.A. Orru, J. Org. Chem. 72,6135 (2007)

13. T.J.J. Müller, Chem. Heterocycl. Comp. 53, 381

(2017)

14. B. Groenendaal, E. Ruijter, R.V.A. Orru, Chem. Commun. 43,5474

(2008)

15. B. Willy, T.J.J. Müller, Org. Lett. 13, 2082

(2011)

16. T.S. Jin, J.S. Zhang, A.Q. Wang, Y.S. Li, Ultrason. Sono Chem. 13,

220 (2006)

17. S. Rostamizadeh, A. AliMohammad, G.H. Mahdavinia, H. Sepehrian, S. Ebrahimia, Synthesis 8,1356 (2010)

18. M. Gholinejad, F. Hamed, C. Nájera, Synlett 27, 1193

(2016)

19. F. Yates, R.T. Courts, A.F. Casy, in Pyridine and Its Derivatives Supplement IV, vol. 445, ed. By R.A. Abramovitch (Wiley, New York, 1975) 
20. R. Vardanyan, V. Hruby, Synthesis of Essentials Drugs, vol. 51 (Elsevier, Amsterdam, 2006)

21. F. Manna, F. Chimenti, A. Bolasco, B. Bizzarri, W. Filippelli, A. Filippelli, L.G. Gagliardi, Eur. J. Med. Chem. 34, 245 (1999)

22. A. Altundas, S. Ayvaz, E. Logoglu, Med. Chem. Res. 20, 1 (2011)

23. Q. Luo, R. Huang, Q. Xiao, Y. Yao, J. Lin, S.-J. Yan, J. Org. Chem. 84, 1999 (2019)

24. X.X. Du, Q.X. Zi, Y.M. Wu, Y. Jin, J. Lin, S.J. Yan, Green Chem. 21, 150 (2019)

25. H. Suga, Y. Furihata, A. Sakamoto, K. Itoh, Y. Okumura, T. Tsuchida, A. Kakehi, T.J. Baba, Org. Chem. 76, 7377 (2011)

26. J.M. DeLosSantos, Y. López, D. Aparicio, F. Palacios, J. Org. Chem. 73, 550 (2008)

27. W. Yu, Z.W. Zhai, D.E. Wedge, S.O. Duke, H.K. Wu, J.Q. Weng, C.X. Tan, Y.G. Zhang, X.H. Liu, Res ChemIntermed 45, 5989 (2019)

28. Z. Kibou, N. Cheikh, N. Choukchou-Braham, B. Mostefa-Kara, M. Benabdellah, D. Villemin, J. Mater. Environ. Sci. 3, 293 (2011)

29. N. Cheikh, D. Villemin, N. Bar, J.-F. Lohier, N. Choukchou-Braham, B. Mostefa- Kara, J. Sopkova, Tetrahedron 69, 1234 (2013)

30. D. Villemin, N. Cheikh, L. Liao, N. Bar, J.F. Lohier, J. Sopkova, N. Choukchou-Braham, B. Mostefa-Kara, Tetrahedron 68, 4906 (2012)

31. Z. Kibou, N. Cheikh, D. Villemin, N. Choukchou-Braham, B. Mostefa-Kara, M. Benabdallah, Int. J. Org. Chem 1, 242 (2011)

32. A. Berrichi, R. Bachir, S. Bedrane, N. Choukchou-Braham, K. Belkacemi, Res. Chem. Intermed. 45, 3481 (2019)

33. D. Villemin, Z. Belhadj, N. Cheikh, N. Choukchou-Braham, N. Bar, J.F. Lohier, Tetrahedron Lett. 54, 1664 (2013)

34. A. Berrichi, R. Bachir, M. Benabdallah, N. Choukchou-Braham, Tetrahedron Lett. 56, 1302 (2015)

35. M.E. Davis, Nature 417, 813 (2002)

36. S. Izquierdo-Barba, M. Sánchez-Salcedo, M.J. Colilla, M.T. Portolés, M. Vallet-Regí, Acta Biomater. 57, 2977 (2011)

37. B. Boukoussa, N. Aouad, R. Hamacha, A. Bengueddach, J. Phys. Chem. Solids 78, 78 (2015)

38. B. Boukoussa, F. Sebih, R. Hamacha, S. Bellahouel, A. Derdour, A. Bengueddach, Res ChemIntermed. 41, 2221 (2015)

39. K. Chikh, B. Boukoussa, L. Bouhadjar, M. Bencheikh, R. Hamacha, R. Meghabar, M. Belbachir, A. Bengueddach, Res ChemIntermed 41, 6485 (2015)

40. A. Hakiki, R.M. Kerbadou, B. Boukoussa, H. HabibZahmani, F. Launay, A. Pailleret, F. Pillier, S. Hacini, A. Bengueddach, R. Hamacha, J. Inorgan. Organometall. Polym. Mater. 29, 1773 (2019)

41. B. Boukoussa, A. Hakiki, N. Bouazizi, A.P. Beltrao-Nunes, F. Launay, A. Pailleret, F. Pillier, A. Bengueddach, R. Hamacha, A. Azzouz, J. Mol. Struct. 1191, 175 (2019)

42. A. Hakiki, B. Boukoussa, H. HabibZahmani, R. Hamacha, N.E.H. HadjAbdelkader, F. Bekkar, F. Bettahar, A.P. Nunes-Beltrao, S. Hacini, A. Bengueddach, A. Azzouz, Mater. Chem. Phys. 212, 415 (2018)

43. B. Boukoussa, R. Hamacha, A. Morsli, A. Bengueddach, Arab. J. Chem. 10, S2160 (2017)

44. B. Boukoussa, A. Hakiki, S. Moulai, K. Chikh, D.E. Kherroub, L. Bouhadjar, D. Guedal, K. Messaoudi, F. Mokhtar, R. Hamacha, J. Mater. Sci. 53, 7372 (2018)

45. I. Terrab, R. Ouargli, B. Boukoussa, K. Ghomari, R. Hamacha, R. Roy, A. Azzouz, A. Bengueddach, Res. Chem. Intermed. 43, 3775 (2017)

46. Z. Abid, A. Hakiki, B. Boukoussa, F. Launay, H. Hamaizi, A. Bengueddach, R. Hamacha, J. Mater. Sci. 54, 7679 (2019)

47. X. Liu, H. Sun, Y. Yang, J. Colloid Interface Sci. 319, 377 (2008)

48. P.R. Selvakannan, K. Mantri, J. Tardio, S.K. Bhargava, J. Colloid Interface Sci. 394, 475 (2013)

49. K.R. Kloetstra, M. VanLaren, H. VanBekkum, J. Chem. Soc., Faraday Trans. 93, 1211 (1997)

50. I. Sobczak, M. Ziolek, E. Pérez-Mayoral, D. Blasco-Jiménez, A. López-Peinado, J. MartínAranda, Catal. Today 179, 159 (2012)

51. S. Roy, T. Chatterjee, M. Pramanik, A.S. Ro, A. Bhaumik, S.K. Manirul Islam, J. Mol. Catal. A: Chem. 386, 78 (2014 


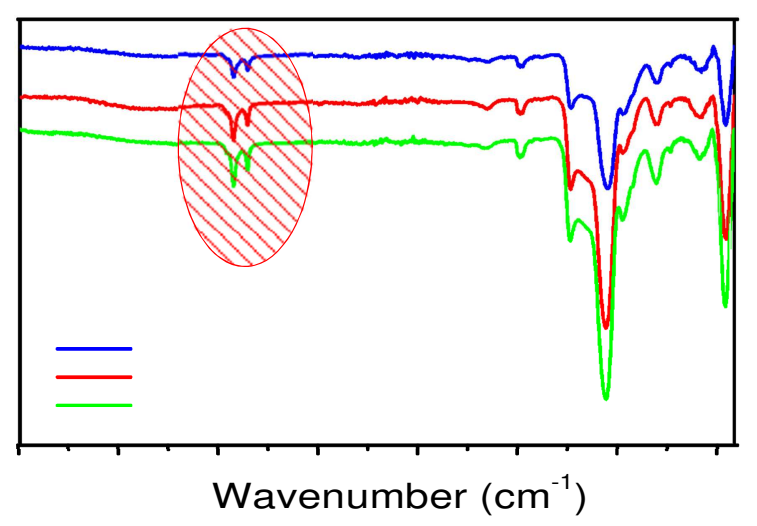

Fig. 1 FTIR spectra of as-synthesized Si-MCM-41 and its modified counterparts

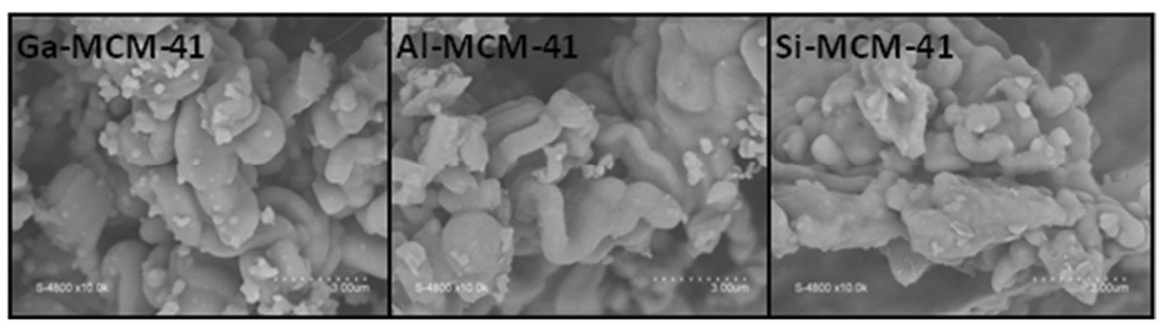

Fig. 2 SEM images of as-synthesized Si-MCM-41 and its modified counterparts 


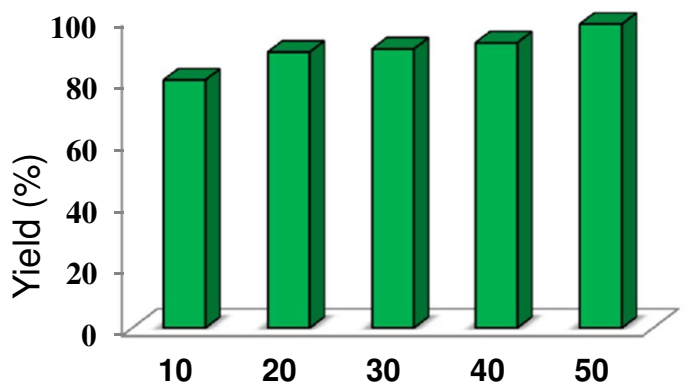

Fig. 3 Effect of the

catalyst amount (as-

synthesized Si- MCM-

41) on the yield of $5 a$

$\mathbf{m o l} \% \mathbf{m o l} \% \mathbf{m o l} \% \mathbf{m o l} \% \mathbf{m o l} \%$

amount of catalysts 


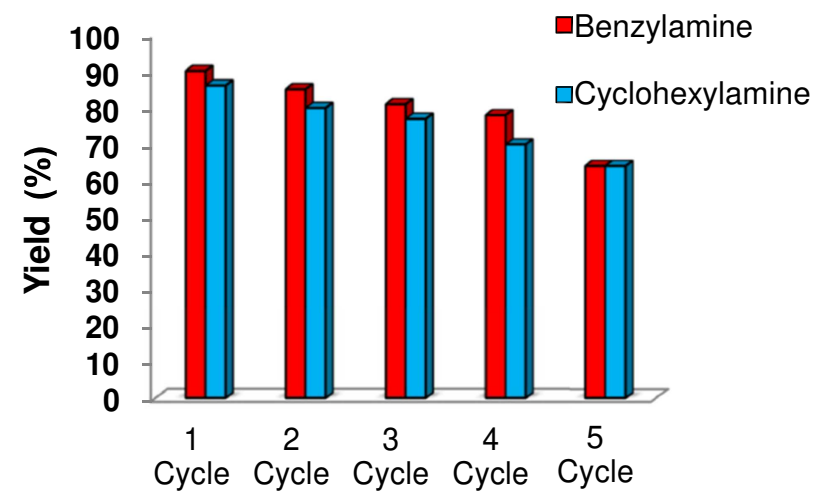

Fig. 4 Catalyst reusability 
Table 1: Four-component reaction of 2-aminopyridines derivatives without catalyst

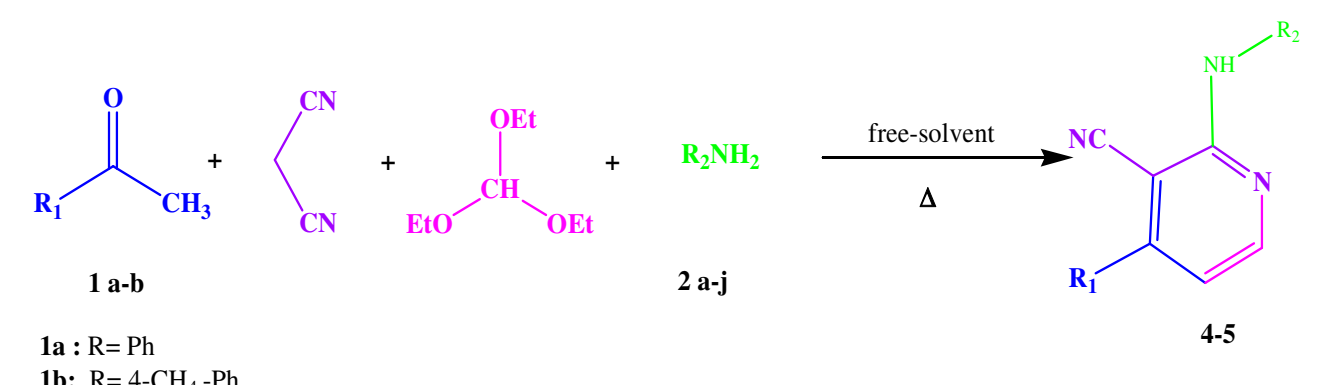

1b: $\mathrm{R}=4-\mathrm{CH}_{4}-\mathrm{Ph}$

\begin{tabular}{|c|c|c|c|c|}
\hline Entry & R1 & R2 & Product & Yield (\%) \\
\hline 1 & & Benzyl- & $4 a_{a}$ & 73 \\
\hline 2 & & Cyclohexyl- & $4_{b}$ & 68 \\
\hline 3 & $\mathrm{C}_{6} \mathrm{H}_{5^{-}}$ & Hexyl- & $4_{c}$ & 70 \\
\hline 4 & & Propyl- & $4_{d}$ & 62 \\
\hline 5 & & Benzyl- & $5 \mathrm{a}$ & 76 \\
\hline 6 & & Cyclohexyl- & $5_{b}$ & 65 \\
\hline 7 & $\mathrm{p}-\mathrm{CH}_{3} \mathrm{C}_{6} \mathrm{H}_{4-}$ & Hexyl- & 5 & 72 \\
\hline 8 & & Propyl- & $5_{d}$ & 66 \\
\hline
\end{tabular}




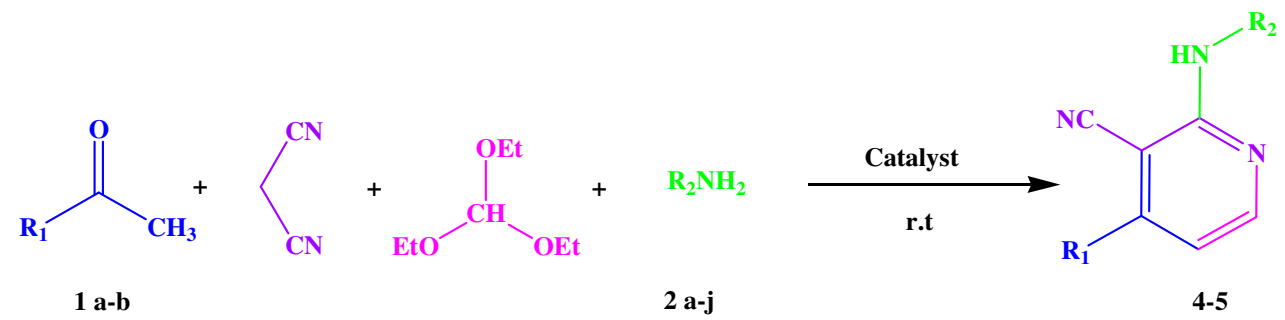

1a: $\mathrm{R}=\mathrm{Ph}$

1b: $\mathrm{R}=4-\mathrm{CH}_{4}-\mathrm{Ph}$

Scheme.1. Four-component reaction of 2-aminopyridines derivatives

Table.2. Optimization of the reaction conditions in the synthesis of $\mathbf{4 a}$

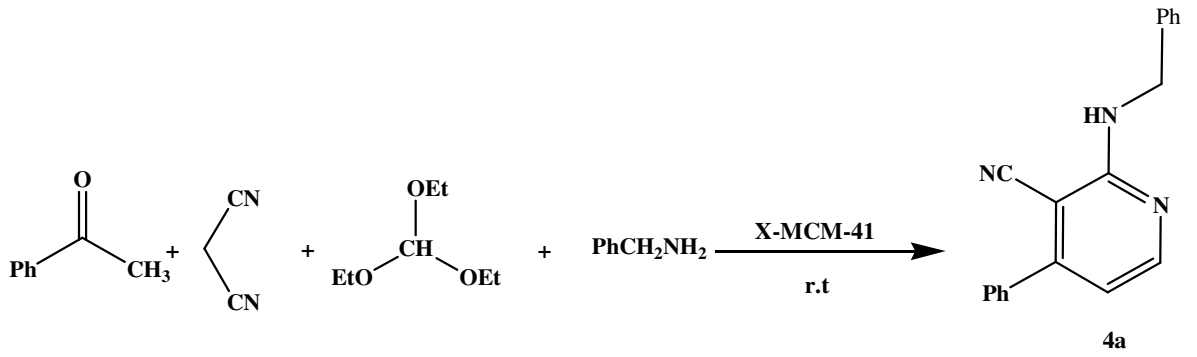

$\mathrm{X}: \mathrm{Ga}, \mathrm{Al}$ or $\mathrm{Si}$

\begin{tabular}{llll}
\hline Entry & Catalyst & Time $(\mathbf{m i n})$ & Yield (\%) \\
\hline $\mathbf{1}$ & Al-MCM-41 & 30 & 67 \\
$\mathbf{2}$ & Ga-MCM-41 & 20 & 75 \\
$\mathbf{3}$ & Si-MCM-41 & 15 & 89 \\
\hline
\end{tabular}


Table.3. Synthesis of 2-aminopyridines catalyzed by Al- Ga or Si-MCM-41 under solvent free-conditions

\begin{tabular}{|c|c|c|c|c|c|c|}
\hline Entry & $\mathbf{R}_{1}$ & $\mathbf{R}_{2}$ & Catalyst & Product & Time(min) & Yield (\%) \\
\hline 1 & \multirow{4}{*}{$\mathrm{C}_{6} \mathrm{H}_{5^{-}}$} & Benzyl- & \multirow{4}{*}{ Al-MCM-41 } & $4 a_{a}$ & 30 & 67 \\
\hline 2 & & Cyclohexyl- & & $4_{b}$ & 35 & 70 \\
\hline 3 & & Hexyl- & & $4_{c}$ & 40 & 73 \\
\hline 4 & & Propyl- & & $4_{d}$ & 50 & 70 \\
\hline 5 & \multirow{4}{*}{$\mathrm{C}_{6} \mathrm{H}_{5^{-}}$} & Benzyl- & \multirow{4}{*}{ Ga-MCM-41 } & $4 a$ & 28 & 75 \\
\hline 6 & & Cyclohexyl- & & $4_{b}$ & 30 & 79 \\
\hline 7 & & Hexyl- & & $4_{c}$ & 38 & 80 \\
\hline 8 & & Propyl- & & $4_{d}$ & 45 & 73 \\
\hline 9 & \multirow{4}{*}{$\mathrm{C}_{6} \mathrm{H}_{5^{-}}$} & Benzyl- & \multirow{4}{*}{ Si-MCM-41 } & $4 a$ & 15 & 89 \\
\hline 10 & & Cyclohexyl- & & $4_{b}$ & 20 & 80 \\
\hline 11 & & Hexyl- & & $4_{c}$ & 25 & 82 \\
\hline 12 & & Propyl- & & $4_{d}$ & 30 & 75 \\
\hline 13 & \multirow{4}{*}{$\mathrm{p}-\mathrm{CH}_{3} \mathrm{C}_{6} \mathrm{H}_{4-}$} & Benzyl- & \multirow{4}{*}{ Al-MCM-41 } & $5 a$ & 28 & 70 \\
\hline 14 & & Cyclohexyl- & & $5_{b}$ & 22 & 83 \\
\hline 15 & & Hexyl- & & $5_{\mathrm{c}}$ & 20 & 85 \\
\hline 16 & & Propyl- & & $5_{d}$ & 28 & 79 \\
\hline 17 & \multirow{4}{*}{ p- $\mathrm{CH}_{3} \mathrm{C}_{6} \mathrm{H}_{4}-$} & Benzyl- & \multirow{4}{*}{ Ga-MCM-41 } & $5 a$ & 22 & 78 \\
\hline 18 & & Cyclohexyl- & & $5 \mathrm{~b}$ & 20 & 80 \\
\hline 19 & & Hexyl- & & $5_{\mathrm{c}}$ & 17 & 82 \\
\hline 20 & & Propyl- & & $5_{d}$ & 25 & 75 \\
\hline 21 & \multirow{4}{*}{$\mathrm{p}-\mathrm{CH}_{3} \mathrm{C}_{6} \mathrm{H}_{4-}$} & Benzyl- & \multirow{4}{*}{ Si-MCM-41 } & $5 \mathrm{a}$ & 13 & 92 \\
\hline 22 & & Cyclohexyl- & & $5_{b}^{a}$ & 18 & 88 \\
\hline 23 & & Hexyl- & & $5_{c}$ & 20 & 85 \\
\hline 24 & & Propyl- & & $5 \mathrm{~d}$ & 25 & 80 \\
\hline
\end{tabular}

